\title{
Assessment of subjective oral factors associated with self-reported halitosis among adults
}

\begin{abstract}
Halitosis is the unpleasant breath emitted from a person's mouth regardless of whether the odorous substances in the breath originate from oral or non-oral sources.

Purpose: to assess the relation of subjective oral factors and self-reported halitosis among adults living in Riyadh, Saudi Arabia.

Materials and methods: A self-administered questionnaire was distributed to 3000 adults. The sample was randomly and equally selected from the five locations in Riyadh (east, west, north, south and center). Data was statistically analyzed using Chi square test at $95 \%$ confidence $(p<0.05)$

Results: Self-reported halitosis was found to be significantly related statistically, to the presence of dental caries, bleeding gingiva, painful gingiva, sensitive teeth, stained teeth, tongue coating, dry mouth, mouth ulcerations, difficulty in chewing food, missing teeth, problematic wisdom teeth, and perceived taste disturbance $(\mathrm{P}=0.001,0.0001,0.0001$, $0.002,0.0001,0.0001,0.0001,0.002,0.0001,0.0001,0.001$ and 0.0001 , respectively). Whereas, there was no statistically significant relation between self-reported halitosis and the presence of orthodontic appliances $(\mathrm{P}=0.469)$. In general, self-reported halitosis was highly significantly related to the frequency of visiting the dentist $(\mathrm{P}=0.0001)$.
\end{abstract}

Conclusion: Oral factors, including dental caries, gingivitis, stained teeth, sensitive teeth, tongue coatings, dry mouth, mouth ulcerations, missing teeth and problematic wisdoms may be associated with self-reported halitosis among adults living in Riyadh.

Keywords: self-reported halitosis, oral factors, dental visits, riyadh

\author{
Volume 5 Issue 6 - 2016
}

\author{
Salwa Al Sadhan,' Amel Darwish ${ }^{2}$ \\ 'Department of Periodontics and Community Dentistry, King \\ Saud University, Saudi Arabia \\ ${ }^{2}$ Department of Pediatric and Community Dentistry, Alexandria \\ University, Egypt
}

\begin{abstract}
Correspondence: Amel Darwish, Department of Pediatric and Community Dentistry, Alexandria University, Champilion Street, Alexandria, Egypt, Tel 002-03-4869690, Email amel_g_darwish@hotmail.com
\end{abstract}

Received: December 08, 2016 | Published: December 16 2016

\section{Introduction}

Halitosis is the unpleasant breath emitted from a person's mouth regardless of whether the odorous substances in the breath originate from oral or non-oral sources. ${ }^{1}$ Halitosis may play an important role in social communication and, therefore, may be the origin of concern not only for a possible health condition but also for frequent psychological alterations leading to social and personal isolation. ${ }^{2}$ It is now widely accepted that the primary cause of halitosis is the release of volatile sulphur compounds (VSCs), which include hydrogen sulphide, dimethyl sulphide, and methyl mercaptan. ${ }^{3}$ These compounds result from the proteolytic degradation by predominantly anaerobic Gramnegative oral microorganisms of various sulphur-containing substrates in food debris, saliva, blood, and epithelial cells. ${ }^{4}$ Van den Broek et al., ${ }^{5}$ concluded that in $90 \%$ of cases, the causes of halitosis are located in the mouth and can be related to deep carious lesions, periodontal disease, oral infections, peri-implant disease, peri-coronitis, mucosal ulcerations, impacted food or debris, factors causing decreased salivary flow rate and tongue coating. ${ }^{5}$ Also, Nalcaci et al., ${ }^{6}$ found that the factors most strongly associated with self-reported halitosis and perceived taste disturbance were subjective oral dryness, tongue coating, inadequate oral hygiene practice, smoking and partial and/ or complete denture wear. Patients with periodontal diseases were found to have deep periodontal pockets that are associated with increased levels of VSCs. ${ }^{7,8}$ Not only the periodontal pockets, but also the presence of active periodontal inflammation was suggested to be more important for the production of oral malodour. ${ }^{9}$ Recent studies showed that tongue coating was one of the most important factors causing halitosis. ${ }^{10,11} \mathrm{Bad}$ breath was also found to be correlated to oral hygiene and dental visits. ${ }^{12,13}$ The aim of this study is to assess the relation of subjective oral factors and self-reported halitosis among adults living in Riyadh, Saudi Arabia.

\section{Materials and methods}

This descriptive, cross-sectional study was ethically approved by College of Dentistry Research Center, King Saud University, Riyadh, Saudi Arabia. This research center is an institutional review board that has an ethics committee that handles the ethical issues concerning the research studies. The study was performed in accordance with the Declaration of Helsinki. The purpose and objectives of the study were stated at the beginning of the questionnaire and answering the questionnaire was considered as consent to participate. Three thousand subjects were randomly selected. The sample included equal groups of school students, college students and employees, and within each group, male and female subjects were equally represented. The sample was randomly and equally selected from the five locations in Riyadh, Saudi Arabia (east, west, north, south and center). A specially designed self-administered questionnaire, developed in English then translated to Arabic, was distributed to the selected research subjects. An expert faculty member reviewed the questionnaire which was then modified according to her comments to ensure the questionnaire validity. The questionnaire began with introductory information regarding "Halitosis" then a statement indicating that the participants' answers would be stored and transmitted securely for confidentiality. At first, the questionnaire included questions concerning the participants' socio-demographic factors. Then, the questionnaire inquired about the participant's perception of any malodor (halitosis) and its history. Finally, it inquired if the subject visits the dentist and if he/she suffers from dental caries, bleeding or tender gingiva, sensitive teeth, dry mouth, oral ulcerations, difficulty in chewing, missing teeth, problems 
with wisdom tooth, taste disturbance and finally the questionnaire inquired if the participant has any orthodontic appliances.

\section{Statistical analysis}

Data was analyzed using Statistical Package for Social Sciences program for Windows (version 16 SPSS Inc., Chicago, IL, USA). Means and frequency distributions were calculated and Chi square test at $95 \%$ confidence $(p<0.05)$ was used to calculate relations of the variables with self-reported halitosis.

\section{Results}

Out of the 3000 questionnaires distributed, 2343 ones were collected giving a response rate of $78.1 \%$. Socio-demographic characteristics of the study sample are presented in Table 1. About two thirds of the sample was within the 17-24 years old age group and the male participants consisted $46.6 \%$ of the sample. Regarding occupation, high-school students, college students and employees represented $38.7 \%, 27.2 \%$ and $34.1 \%$ of the sample, respectively. The relation of self-reported halitosis and subjective oral factors among adults living in Riyadh is presented in Table 2. Self-reported halitosis was found to be significantly related statistically, to the presence of dental caries $(\mathrm{P}=0.001)$, bleeding gingiva $(\mathrm{P}=0.0001)$, painful gingiva $(\mathrm{P}=0.0001)$, sensitive teeth $(\mathrm{P}=0.002)$, stained teeth $(\mathrm{P}=0.0001)$, tongue coating $(\mathrm{P}=0.0001)$, dry mouth $(\mathrm{P}=0.0001)$, mouth ulcerations $(\mathrm{P}=0.002)$, difficulty in chewing food $(\mathrm{P}=0.0001)$, missing teeth $(\mathrm{P}=0.0001)$, problematic wisdom teeth $(\mathrm{P}=0.001)$, and perceived taste disturbance $(\mathrm{P}=0.0001)$. Whereas, there was no statistically significant relation between self-reported halitosis and the presence of orthodontic appliances $(\mathrm{P}=0.469)$. In general, self-reported halitosis was highly significantly related to the frequency of visiting the dentist $(\mathrm{P}=0.0001)$ (Figure 1).

Table I Socio-demographic characteristics of the study sample $(n=2343)$

\begin{tabular}{|c|c|}
\hline Variable & Frequency; n (\%) \\
\hline \multicolumn{2}{|l|}{ Age } \\
\hline 17-24 years & I559 (66.7) \\
\hline $25-34$ years & $436(18.6)$ \\
\hline $35-44$ years & $190(8.1)$ \\
\hline $45-54$ years & II $3(4.8)$ \\
\hline$>54$ years & $40(1.7)$ \\
\hline \multicolumn{2}{|l|}{ Gender } \\
\hline Male & $1093(46.6)$ \\
\hline Female & $1250(53.4)$ \\
\hline \multicolumn{2}{|l|}{ Education level } \\
\hline Illiterate & $8(0.3)$ \\
\hline$<$ High school & $915(39.1)$ \\
\hline $\begin{array}{l}\text { High school-<Bachelor } \\
\text { degree }\end{array}$ & $1292(55.1)$ \\
\hline Post graduate & III (4.7) \\
\hline \multicolumn{2}{|l|}{ Occupation } \\
\hline High school students & $907(38.7)$ \\
\hline College students & $638(27.2)$ \\
\hline Employee & $798(34.1)$ \\
\hline \multicolumn{2}{|l|}{ Self-reported halitosis } \\
\hline Yes & $534(22.8)$ \\
\hline No & 1809 (77.2) \\
\hline
\end{tabular}

Table 2 Relation of self-reported halitosis and oral factors among the study population

\begin{tabular}{|c|c|c|}
\hline Variable & $\begin{array}{l}\text { Subjects With Halitosis, } \\
\text { n (\%) }\end{array}$ & X2* (P value) \\
\hline \multicolumn{3}{|c|}{ Dental caries } \\
\hline Yes & $305(57.3)$ & $9.165(0.001) \dagger$ \\
\hline \multicolumn{3}{|c|}{ Bleeding gingiva } \\
\hline Yes & $166(32)$ & $65.04(0.0001) \dagger$ \\
\hline \multicolumn{3}{|c|}{ Painful gingiva } \\
\hline Yes & $133(25.4)$ & $44.196(0.0001) \dagger$ \\
\hline \multicolumn{3}{|c|}{ Sensitive teeth } \\
\hline Yes & $177(33.8)$ & $8.832(0.002) \dagger$ \\
\hline \multicolumn{3}{|c|}{ Stained teeth } \\
\hline Yes & $164(3 \mid .3)$ & $16.741(0.0001) \dagger$ \\
\hline \multicolumn{3}{|c|}{ Tongue coating } \\
\hline Yes & $92(17.7)$ & $51.882(0.0001) \dagger$ \\
\hline \multicolumn{3}{|c|}{ Dry mouth } \\
\hline Yes & $147(27.5)$ & $84.675(0.0001) \dagger$ \\
\hline \multicolumn{3}{|c|}{ Mouth ulcerations } \\
\hline Yes & $43(8.1)$ & $9.358(0.002) \dagger$ \\
\hline \multicolumn{3}{|c|}{$\begin{array}{l}\text { Difficulty in chewing } \\
\text { food }\end{array}$} \\
\hline Yes & $60(11.3)$ & $31.978(0.0001) \dagger$ \\
\hline \multicolumn{3}{|c|}{ Missing teeth } \\
\hline Yes & $185(34.7)$ & $14.942(0.0001) \dagger$ \\
\hline \multicolumn{3}{|c|}{$\begin{array}{l}\text { Problematic wisdom } \\
\text { teeth }\end{array}$} \\
\hline Yes & $146(27.4)$ & $9.573(0.001) \dagger$ \\
\hline \multicolumn{3}{|c|}{$\begin{array}{l}\text { Perceived taste } \\
\text { disturbance }\end{array}$} \\
\hline Yes & $168(31.7)$ & $\begin{array}{l}186.756(0.0001) \\
\dagger\end{array}$ \\
\hline \multicolumn{3}{|c|}{ Ortho appliances } \\
\hline Yes & $66(12.5)$ & $0.027(0.469)$ \\
\hline \multicolumn{3}{|c|}{ Visiting the dentist } \\
\hline Yes & $450(85.4)$ & $20.244(0.0001) \dagger$ \\
\hline
\end{tabular}

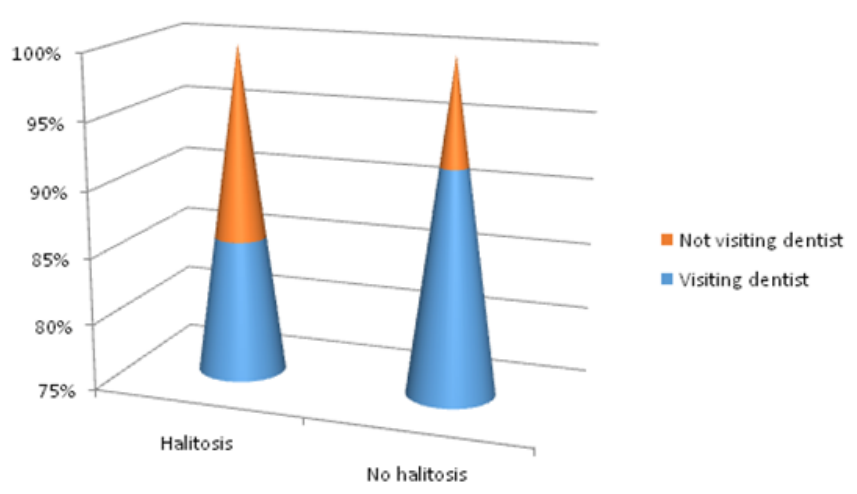

Figure I Relation of self-reported halitosis and visiting the dentist among the study sample. 


\section{Discussion}

Most of the oral factors can have a great role in causing halitosis due to tissue breakdown, putrefaction of amino acids and decreasing of saliva flow. All these conditions result in the release of volatile sulphur compounds (VSC). ${ }^{14,15}$ There was a statistically significant relation between dental caries and halitosis. This agrees with Nalcaci et al. ${ }^{16}$ who found that caries experience was associated with malodor among children. This study found that there were statistically significant relations between self-reported halitosis and bleeding and painful gingiva. Some microorganisms in periodontal lesions of gingivitis and periodontitis can produce large amounts of volatile sulphur compounds. ${ }^{4}$ The study also found that subjective tongue coating has a significant relation to self-reported halitosis. As a consequence of the tongue's large and papillary surface area, the dorsum of the tongue can retain large amounts of desquamated cells, leucocytes, and microorganisms. A study conducted in Japan correlated periodontitis and tongue coating to VSC scores. ${ }^{9}$ Also, severe periodontitis patients presented higher halitosis scores than non-periodontal patients. In two studies carried out in Switzerland, tongue coating was considered an influencing factor for halitosis. ${ }^{17,18}$ Smoking and periodontal diseases were also associated with higher halitosis rankings. ${ }^{17}$ A statistically significant relation was found between subjective dry mouth and self-reported halitosis. This agrees with the findings of Albuquerque et al., ${ }^{19}$ who reported that patients with a dry mouth often showed an increased volume of plaque on teeth and tongue. ${ }^{19}$ The lack of salivary flow, leads to the disappearance of the antimicrobial activity of the saliva and the transition from Gram-positive bacteria to Gram-negative species. ${ }^{20}$ Other studies described accurately the correlation between dry mouth and the increase of halitosis. ${ }^{21,22} \mathrm{~A}$ statistically significant relation was found between mouth ulcerations and problematic wisdom teeth and self-reported halitosis. This agrees with a study that described pericoronitis, recurrent oral ulcerations and herpetic gingivitis as origin for bad breath. ${ }^{23}$ It was observed that difficulty in chewing and missing teeth are statistically related to self-reported halitosis. This may be because both factors result in accumulation and putrefaction of food remnants which contribute to bad breath. No statistically significant relationship was found between the presence of orthodontic appliances and self-reported halitosis. This may be because people who have orthodontic appliances for correction of their teeth mal-alignment can be enthusiastic to perform the proper oral hygiene instructions which help them to avoid having oral malodor. Visiting the dental clinic was found to have a significant statistical relation to self-reported halitosis. This agrees with a study in Sweden and another in Japan that showed that calculus, plaque and limited dental visits were significantly correlated to severe halitosis. ${ }^{12,13}$ This study was cross-sectional, which did not allow the examination of cause-and-effect relations. Further analytical studies are recommended to be conducted to assess the association of halitosis and oral factors.

\section{Conclusion}

It can be concluded that oral factors, including dental caries, gingivitis, stained teeth, sensitive teeth, tongue coatings, dry mouth, mouth ulcerations, missing teeth and problematic wisdoms may be associated with self-reported halitosis among adults living in Riyadh. Dentists are the first line professionals to be confronted with this problem. They should be educated about the origin, detection and the treatment of this pathology. Educating the public about the causes of halitosis can also help in the management of this problem, as periodic visits to the dental clinic for assessment and treatment of any oral factor that may cause halitosis, can also lead to the amelioration of this problem.

\section{Acknowledgments}

None.

\section{Conflicts of interest}

The authors declare that there are no conflicts of interest.

\section{Funding details}

None.

\section{References}

1. Cortelli JR, Barbosa MDS, Westphal MA. Halitosis:a review of associated factors and therapeutic approach. Braz Oral Res. 2008;22(1):44-54.

2. Sanz M, Roldan S, Herrera D. Fundamentals of Breath Malodour. $J$ Contemp Dent Pract. 2001;2(4):1-17.

3. Lee PPC, Mak WY, Newsome P. The etiology and treatment of oral halitosis:an update. Hong Kong Med J. 2004;10(6):414-418.

4. Tonzetich J. Production and origin of oral malodor:a review of mechanisms and methods of analysis. J Periodontol. 1977;48(1):13-20.

5. Van den Broek AM, Feenstra L, De Baat C. A review of the current literature on management of halitosis. Oral Dis. 2008;14(1):30-39.

6. Nalcaci R, Baran I. Factors associated with self-reported halitosis (SRH) and perceived taste disturbance (PTD) in elderly. Arch Gerontol Geriatr. 2008;46(3):307-316.

7. Coli JM, Tonzetich J. Characterization of volatile sulphur compounds production at individual gingival crevicular sites in humans. J Clin Dent. 1992;3(4):97-103.

8. Yaegaki K, Sanada K. Biochemical and clinical factors influencing oral malodor in periodontal patients. J Periodontol. 1992;63(9):783-789.

9. Miyazaki H, Sakao S, Katoh Y, et al. Correlation between volatile sulphur compounds and certain oral health measurements in the general population. J Periodontol. 1995;66(8):679-684.

10. Pham TA, Ueno M, Shinada K, et al. Factors affecting oral malodor in periodontitis and gingivitis patients. $J$ Investig Clin Dent. 2012;3(4):284-290.

11. Lu HX, Tang C, Chen X, et al. Characteristics of patients complaining of halitosis and factors associated with halitosis. Oral dis. 2014;20(8):787-795.

12. Söder B, Johansson B, Söder PO. The relation between foetor ex ore, oral hygiene and periodontal disease. Swed Dent J. 2000;24(3):73-82.

13. Yokoyama S, Ohnuki M, Shinada K, et al. Oral malodor and related factors in Japanese senior high school students. $J$ Sch Health. 2010;80(7):346-352.

14. Delanghe G, Ghyselen J, Bollen C, et al. An inventory of patients' response to treatment at a multidisciplinary breath odor clinic. Quintessence Int. 1990;30(5):307-310.

15. Van Steenberghe D. Breath Malodor a step-by-step approach. (1st edn), Quintessence, Copenhagen, Denmark, Europe, 2004.

16. Nalçaci R, Dülgergil T, Oba AA, et al. Prevalence of breath malodour in 7-11-year-old children living in Middle Anatolia, Turkey. Community Dent Health. 2008;25(3):173-177. 
17. Bornstein MM, Kislig K, Hoti BB, et al. Prevalence of halitosis in the population of the city of Bern, Switzerland:a study comparing selfreported and clinical data. Eur J Oral Sci. 2009;117(3):261-267.

18. Bornstein MM, Stocker BL, Seemann R, et al. Prevalence of halitosis in young male adults:a study in swiss army recruits comparing self-reported and clinical data. J Periodontol. 2009;80(1):24-31.

19. Albuquerque DF, De Souza Tolentino E, Amado FM, et al. Evaluation of halitosis and sialometry in patients submitted to head and neck radiotherapy. Med Oral Pathol Oral Cir Bucal. 2010;15(6):e850-e854.

20. Almstahl A and Wikstrom M. Oral microflora in subjects with reduced salivary secretion. J Dent Res. 1999;78(8):1410-1416.
21. Kleinberg I, Wolff MS, Codipilly DM. Role of saliva in oral dryness, oral feel and oral malodour. Int Dent J. 2002;52(Suppl 3):236-240.

22. Koshimune S, Awano S, Gohara K, et al. Low salivary flow and volatile sulphur compounds in mouth air. Oral Surg Oral Med Oral Pathol Oral Radiol Endod. 2003;9(1):38-41.

23. Delanghe G, Ghyselen J, Van Steenberghe D, et al. Multidisciplinary breath-odour clinic. Lancet. 1997;350(9072):187. 